\title{
A global quantitative survey of hemostatic assessment in postpartum hemorrhage and experience with associated bleeding disorders
}

This article was published in the following Dove Press journal:

International Journal of Women's Health

3 July 2017

Number of times this article has been viewed

\author{
Andra $\mathrm{H}$ James' \\ David L Cooper ${ }^{2}$ \\ Michael J Paidas ${ }^{3}$ \\ 'Department of Obstetrics and \\ Gynecology, Division of Maternal- \\ Fetal Medicine, Duke University, \\ Durham, NC, ${ }^{2}$ Clinical, Medical and \\ Regulatory Affairs, Novo Nordisk \\ Inc., Plainsboro, NJ, ${ }^{3}$ Department \\ of Obstetrics, Gynecology, and \\ Reproductive Sciences, Yale Women \\ and Children's Center for Blood \\ Disorders and Preeclampsia \\ Advancement, Yale University, \\ New Haven, CT, USA
}

Purpose: Coagulopathy may be a serious complicating or contributing factor to postpartum hemorrhage ( $\mathrm{PPH})$, and should be promptly recognized to ensure proper bleeding management. This study aims to evaluate the approaches of obstetrician-gynecologists worldwide towards assessing massive PPH caused by underlying bleeding disorders.

Methods: A quantitative survey was completed by 302 obstetrician-gynecologists from 6 countries (the UK, France, Germany, Italy, Spain, and Japan). The survey included questions on the use of hematologic laboratory studies, interpretation of results, laboratory's role in coagulation assessments, and experience with bleeding disorders.

Results: Overall, the most common definitions of "massive" PPH were $>2,000 \mathrm{~mL}(39 \%)$ and $>1,500 \mathrm{~mL}$ (34\%) blood loss. The most common criteria for rechecking a "stat" complete blood count and for performing coagulation studies were a drop in blood pressure $(73 \%)$ and ongoing visible bleeding (78\%), respectively. Laboratory coagulation (prothrombin time/ activated partial thromboplastin time [PT/aPTT]) and factor VIII/IX assays were performed on-site more often than were mixing studies (laboratory coagulation studies, 93\%; factor VIII/ IX assays, 63\%; mixing studies, 22\%). Most commonly consulted sources of additional information were colleagues within one's own specialty $(68 \%)$ and other specialists $(67 \%)$. Most respondents had consulted with a hematologist (78\%; least, Germany [56\%]; greatest, UK [98\%]). The most common reason for not consulting was hematologist unavailability (44\%). The most commonly reported thresholds for concern with PT and APTT were 13 to 20 seconds (36\%) and 30 to 45 seconds (50\%), respectively. Most respondents reported having discovered an underlying bleeding disorder (58\%; least, Japan [35\%]; greatest, Spain [74\%]).

Conclusion: Global survey results highlight similarities and differences between countries in how PPH is assessed and varying levels of obstetrician-gynecologist experience with identification of underlying bleeding disorders and engagement of hematology consultants. Opportunities to improve patient management of PPH associated with bleeding disorders include greater familiarity with interpreting PT/aPTT test results and identification of and consistent consultation with hematologists with relevant expertise.

Keywords: postpartum hemorrhage, acquired hemophilia, bleeding disorders, hematologist, coagulation studies

\section{Introduction}

Although postpartum hemorrhage (PPH) is most often a consequence of obstetric factors (eg, atony) or surgical bleeding, ${ }^{1-6}$ coagulopathy may be a complicating or contributing factor and may impair attempts to control bleeding if not promptly recognized. Types of coagulopathies include dilutional or consumptive states that 
may develop as a consequence of resuscitative measures for massive $\mathrm{PPH},{ }^{4,7}$ regardless of the precipitating cause, and preexisting acquired or congenital coagulation disorders that may underlie immediate or delayed $\mathrm{PPH}^{4}{ }^{4}$ Acquired disorders that may occur during or after pregnancy include quantitative platelet disorders ${ }^{8}$ such as HELLP (hemolysis, elevated liver enzymes, low platelets) and acquired hemophilia, a rare condition that is characterized by alloantibodies against coagulation factors (most often factor VIII) and is associated with pregnancy in $2 \%$ to $29 \%$ of cases. ${ }^{9-14}$ Among congenital coagulation disorders, von Willebrand disease (VWD), hemophilia carriage (factor VIII or IX deficiency), factor XI deficiency, various rare bleeding disorders (eg, fibrinogen, factors II, V, VII, and X deficiencies), and severe platelet function disorders of Glanzmann's thrombasthenia and Bernard-Soulier syndrome are associated with higherthan-expected rates of PPH. ${ }^{15}$ PPH may be notably delayed in both congenital bleeding disorders and acquired hemophilia, with the latter manifesting as late as 117 days after delivery. ${ }^{4,14,15}$ Acquired hemophilia is a hemorrhagic disorder often presenting with diffuse soft tissue bleeding and exhibiting refractoriness to factor VIII therapy, and potentially causing life-threatening bleeding symptoms. ${ }^{9,11,16,17}$ Because of the extreme rarity of this condition, many obstetriciangynecologists may be unfamiliar with the specialized laboratory assessments necessary to confirm the diagnosis.

Prompt diagnosis of acquired and congenital coagulation disorders requires early and consistent hemostatic assessment; however, recent survey data highlight variability in how different specialists in the USA approach the bleeding patient, and the extent to which health care professionals investigate reasons for bleeding rather than focusing only on the location of bleeding. ${ }^{18,19}$ In a report published recently in the International Journal of Women's Health, data were presented from a quantitative survey assessing practice patterns of obstetrician-gynecologists in the USA regarding PPH management, including assessment for contributing coagulopathy. ${ }^{18}$ Nearly all (98\%) of those surveyed reported experience with what they defined as "massive" or "severe" PPH in the preceding 5 years, although responses revealed variability in definitions of massive $\mathrm{PPH}$, when to obtain coagulation tests, how to interpret test results, and in experience with consulting hematology for identified bleeding disorders. Approximately half of the participants had discovered an underlying bleeding disorder in a patient with PPH, yet fewer than half would check coagulation studies for any criteria other than ongoing visible bleeding in the setting of PPH, including refractoriness to pharmacologic and surgical treatments for PPH or hemodynamic perturbations. In addition, the likelihood of hematologist consultation for PPH was low; $42 \%$ of respondents had never consulted a hematologist for this purpose, and $34 \%$ had done so only 1 or 2 times. These findings revealed potential areas for improved practice in managing massive $\mathrm{PPH}$, such as earlier and more consistent assessment and monitoring of coagulation studies and consultation with a hematologist.

Prompted by the results in the USA, here we report the findings of a similar quantitative survey of obstetriciangynecologists from 6 other countries chosen to represent diverse health care systems. This study aimed to identify similarities and differences in the approach of obstetriciangynecologists and other specialists towards assessment of the bleeding patient with $\mathrm{PPH}$, with a specific focus on experience with identification of an underlying congenital or acquired bleeding disorder.

\section{Materials and methods}

Similar to the US survey previously reported, ${ }^{18}$ obstetriciangynecologists were recruited from the UK, France, Germany, Italy, Spain, and Japan. The survey was translated into the local language in each country. Physicians were randomly sampled from consulting group lists of registered physicians, and they had to be actively practicing to be included. Sampled physicians were invited to participate via email and provided with a URL and password for onetime use to the secure survey website. The online survey took $\sim 25$ minutes to complete, was open for 4 weeks, and was closed for each country at 50 responses. Subjects were compensated for their participation, with amounts ranging from \$56 (France, Germany, Japan, and Italy) to \$101 (UK). The survey included questions on the use of specific hematologic laboratory studies, the interpretation of laboratory results, the roles of respondents' on-site laboratories in facilitating those processes, and respondents' experience with discovering underlying bleeding disorders, including acquired hemophilia. This physician survey was exempt from Institutional Review Board approval, as no private or protected information or biological specimens were elicited, and data collection did not allow for linking responses back to participating physicians or any individual patients.

\section{Results}

Survey respondents included 302 obstetrician-gynecologists from a total of 6 countries: the UK $(n=50)$, France $(n=50)$, Germany $(\mathrm{n}=50)$, Italy $(\mathrm{n}=50)$, Spain $(\mathrm{n}=50)$, and Japan $(n=52)$. The majority of respondents were men $(75 \%)$, and 
the overall mean age was 46.2 years. Respondents had spent a mean of 18.9 years in practice. The majority of respondents (60\%) practiced in a hospital-based setting; $27 \%$ practiced in an office and $12 \%$ reported practicing in both hospital-based and office settings.

Overall, the most common definition of "massive" $\mathrm{PPH}$ was $>2,000 \mathrm{~mL}$ of blood loss (39\%); 34\% defined massive
PPH as $>1,500 \mathrm{~mL}$ of blood loss and $23 \%$ as $>1,000 \mathrm{~mL}$. Compared to other countries, respondents from the UK exhibited highest use of the definition $>2,000 \mathrm{~mL}$ of blood loss $(54 \%)$, and those from France reported highest use of the definition $>1,000 \mathrm{~mL}$ of blood loss $(32 \%)$. The reported use of various management strategies for $\mathrm{PPH}$ across regions is shown in Figure 1. Of uterotonic strategies

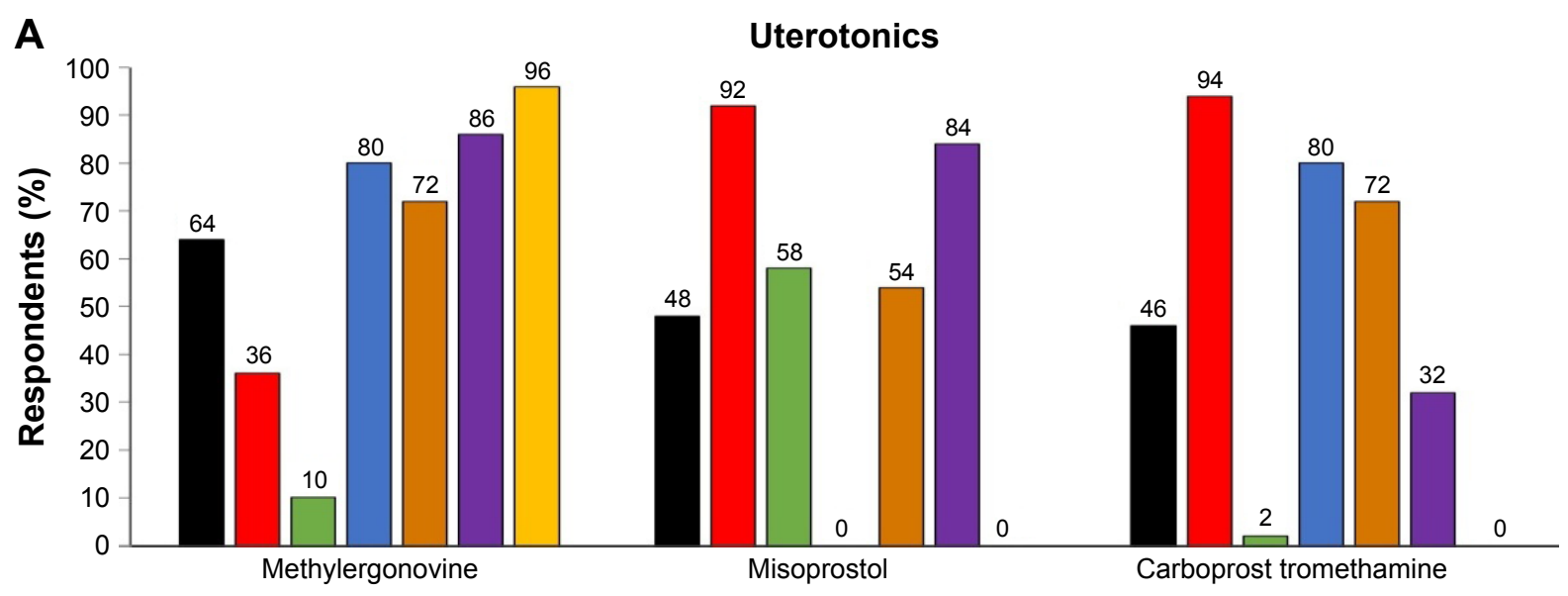

B

Surgery
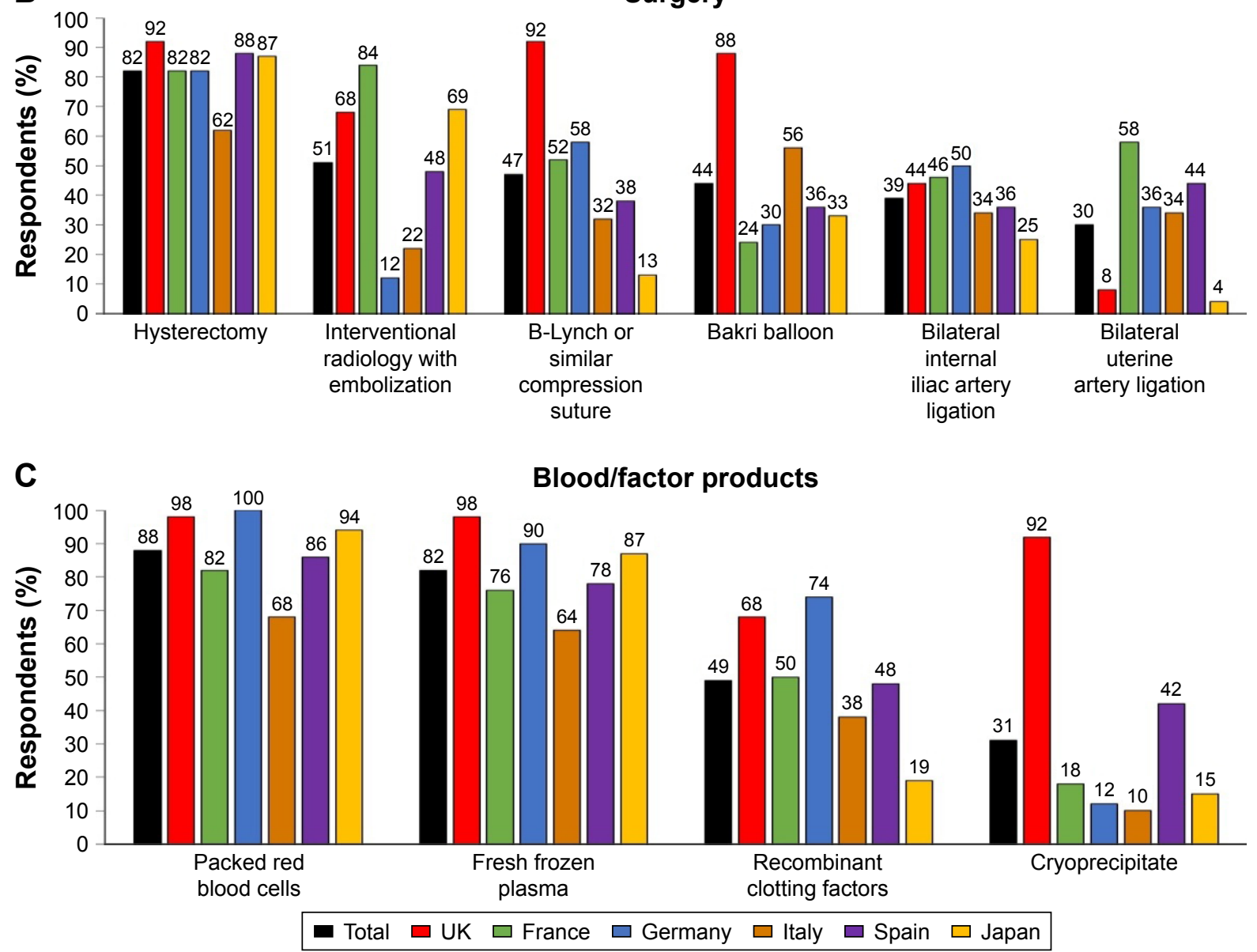

Figure I Strategies used for the management of postpartum hemorrhage: uterotonics (A); surgery (B); and blood/factor products (C). 
(Figure 1A), a majority of respondents reported having used methylergonovine (64\%); fewer respondents had used misoprostol (48\%) or carboprost tromethamine (46\%). Of surgical strategies (Figure 1B), the most commonly reported technique overall was hysterectomy $(82 \%)$. The use of several surgical strategies varied substantially between regions; in particular, reported rates of Bakri balloon and B-Lynch or similar compression suture use were higher in the UK than overall (Bakri balloon, 88\% vs 44\%; B-Lynch or similar compression suture, $92 \%$ vs 47\%), and rates of interventional radiology with embolization were higher in France than overall (84\% vs 51\%). Among blood/factor products (Figure 1C), the most common therapies were packed red blood cells (88\%) and fresh frozen plasma $(82 \%)$.

The most commonly reported criteria for rechecking a "stat" complete blood count were a drop in blood pressure $(73 \%)$, ongoing visible bleeding $(72 \%)$, tachycardia (70\%), and an estimated 1,000 mL of blood loss (63\%); the most common criteria for checking coagulation studies were ongoing visible bleeding (78\%), a drop in hematocrit/ hemoglobin (58\%), and a drop in blood pressure $(57 \%)$. Laboratory coagulation studies and factor VIII/IX studies were most often performed on-site at participants' institutions, rather than being sent to an external lab (coagulation studies, 93\% overall; country-specific responses ranged from $84 \%$ [France] to $98 \%$ [UK and Germany]; factor VIII/IX studies, $63 \%$ overall; country-specific responses ranged from $21 \%$ [Japan] to $76 \%$ [UK]). Mixing studies (in which plasma from an individual without any hemostatic abnormalities is mixed in a 1:1 ratio with plasma from the patient of interest and tested via PT or aPTT to investigate the presence of inhibitors) were less frequently performed on-site (22\% overall; country-specific responses ranged from 10\% [UK] to $44 \%$ [Japan]). In a majority of cases in which coagulation studies had been performed, abnormal results were flagged (overall mean, 71\%; country-specific responses ranged from $42 \%$ [Italy] to $89 \%$ [Germany]). In most cases, normal ranges were also provided for reference (64\% overall).

The resources that respondents most commonly reported consulting for additional information were colleagues within their own specialty (68\%), other specialists (67\%), and PubMed (54\%). Most respondents reported having consulted with a hematologist for PPH (overall, 78\%; country-specific responses ranged from 56\% [Germany] to 98\% [UK]) (Figure 2A); overall, 30\% of respondents reported having consulted with a hematologist 5 or more times (countryspecific responses ranged from 12\% [Germany] to $76 \%$ $[\mathrm{UK}])$. Reported reasons for not consulting with hematology included a hematologist not always being available (44\%), a sense of responsibility towards diagnosing and treating such patients oneself (33\%), comfort with handling (diagnosing and treating) such patients $(20 \%)$, and not having a hematologist on staff at the hospital (19\%) (Figure 2B).

When asked at what level prothrombin time (PT) results would begin to cause concern, most respondents indicated a threshold of 13 to 20 seconds $(36 \%)$ or 21 to 49 seconds (32\%); $7 \%$ indicated a threshold of $<10$ seconds, $6 \%$ indicated 10 to 12 seconds, and $18 \%$ indicated $\geq 50$ seconds (Figure 3A). Of individual countries, Spain was associated with the greatest level of sensitivity to an elevated PT (72\% reported a threshold of $\leq 20$ seconds), and Germany with the lowest sensitivity ( $20 \%$ reported a threshold of $\leq 20$ seconds). When asked at what level activated partial thromboplastin time (aPTT) results would begin to cause concern, most respondents indicated a threshold of 30 to 45 seconds $(50 \%)$ or $<30$ seconds (20\%); $13 \%$ indicated a threshold of 46 to 59 seconds, $14 \%$ indicated 60 to 75 seconds, and $3 \%$ indicated $\geq 76$ seconds (Figure $3 \mathrm{~B}$ ). The UK and Spain were associated with the greatest level of sensitivity to an elevated aPTT ( $82 \%$ of each reported a threshold of $\leq 45$ seconds), and Germany with the lowest sensitivity (52\% reported a threshold of $\leq 45$ seconds). Respondents reported little experience with mixing studies; overall, only $11 \%$ had ordered mixing studies (country-specific responses ranged from $6 \%$ [Italy] to $18 \%$ [Japan]). Respondents also reported little familiarity with mixing studies; on a scale from 1 ("not at all familiar") to 7 ("very familiar"), most respondents indicated a level of 1 to 2 (78\%), and $20 \%$ reported a level of 3 to 5 . Across all countries, Japan was associated with the greatest level of familiarity with mixing studies (52\% reported a level of 3 to 5 and $10 \%$ reported a level of 6 to 7 ).

A majority of respondents reported having discovered an underlying bleeding disorder (58\%); country-specific responses ranged from 35\% (Japan) to 74\% (Spain) (Table 1). A majority (54\%) of respondents were interested in learning more about bleeding disorders; greatest interest was in Spain (70\% rated 6-7 of 7) and UK (66\%), and least in Japan (27\%). Preferred ways to learn more included journals or publications $(54 \%)$, online $(38 \%)$, and national/regional conferences $(33 \%)$.

Among those who had discovered a bleeding disorder, while most probably had encountered disseminated intravascular coagulation or other frequent causes of coagulopathy, exposure to rare disorders was likely more limited. For example, despite post-partum bleeding being cited as a common presentation of acquired hemophilia in women of 
A

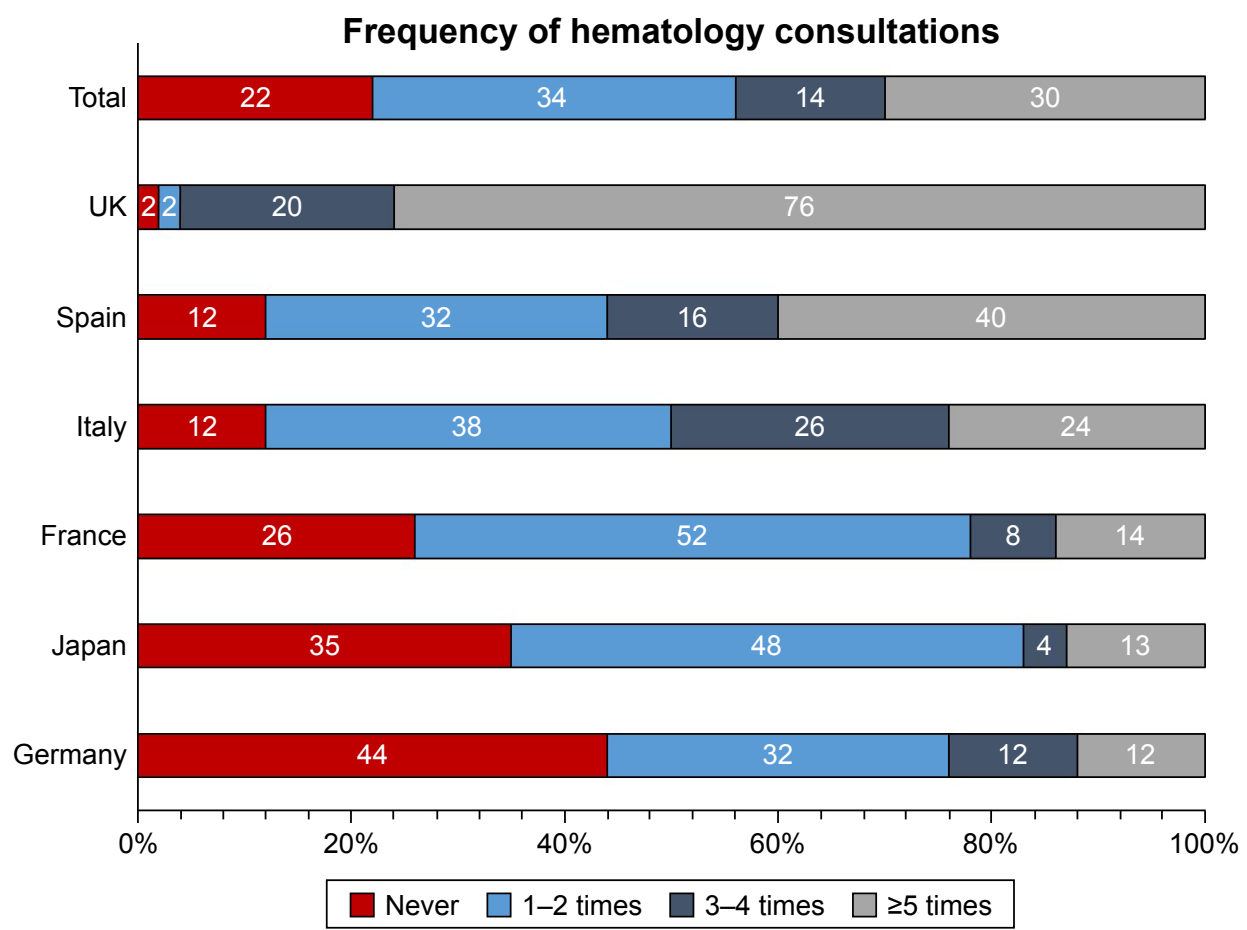

B 70 Reasons for not consulting hematology

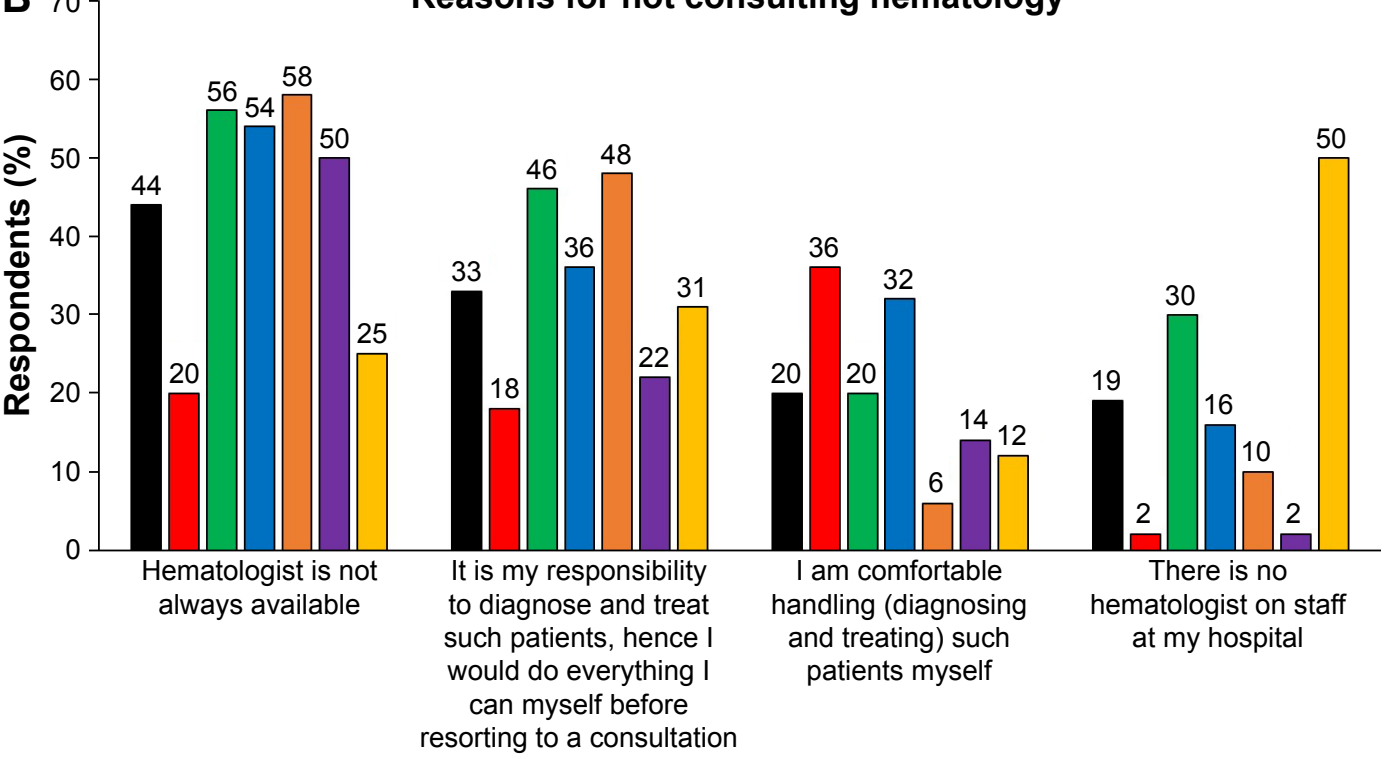

Total $\square$ UK $\square$ France $\square$ Germany $\square$ Italy $\square$ Spain $\square$ Japan

Figure 2 Frequency of hematology consultations (A) and reasons for not consulting hematology (B).

childbearing age, few respondents reported having seen it (overall, 23\% reported encountering acquired hemophilia; country-specific responses ranged from $0 \%$ [Japan] to $43 \%$ [Germany]). Some respondents had never heard of acquired hemophilia or knew the name only (36\%); $20 \%$ reported having some or a lot of knowledge regarding acquired hemophilia.

\section{Discussion}

The results of this global quantitative survey expand upon findings from the previously published survey of US obstetrician-gynecologists by capturing responses across different health care delivery systems. Overall, the global sample was similar to the US sample regarding mean age and mean years in practice. US respondents had a higher 
A

PT thresholds for concern

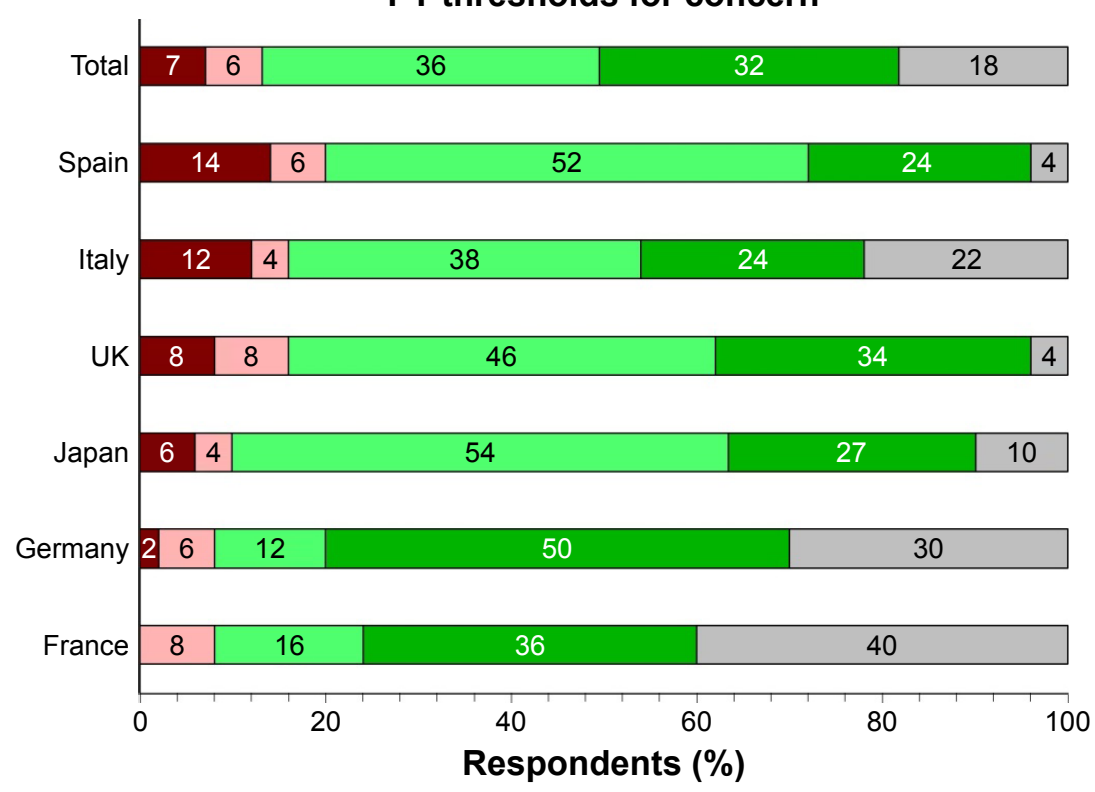

<10 seconds $\square 10-12$ seconds $\square 13-20$ seconds $\square 21-49$ seconds $\square \geq 50$ seconds

\section{B}

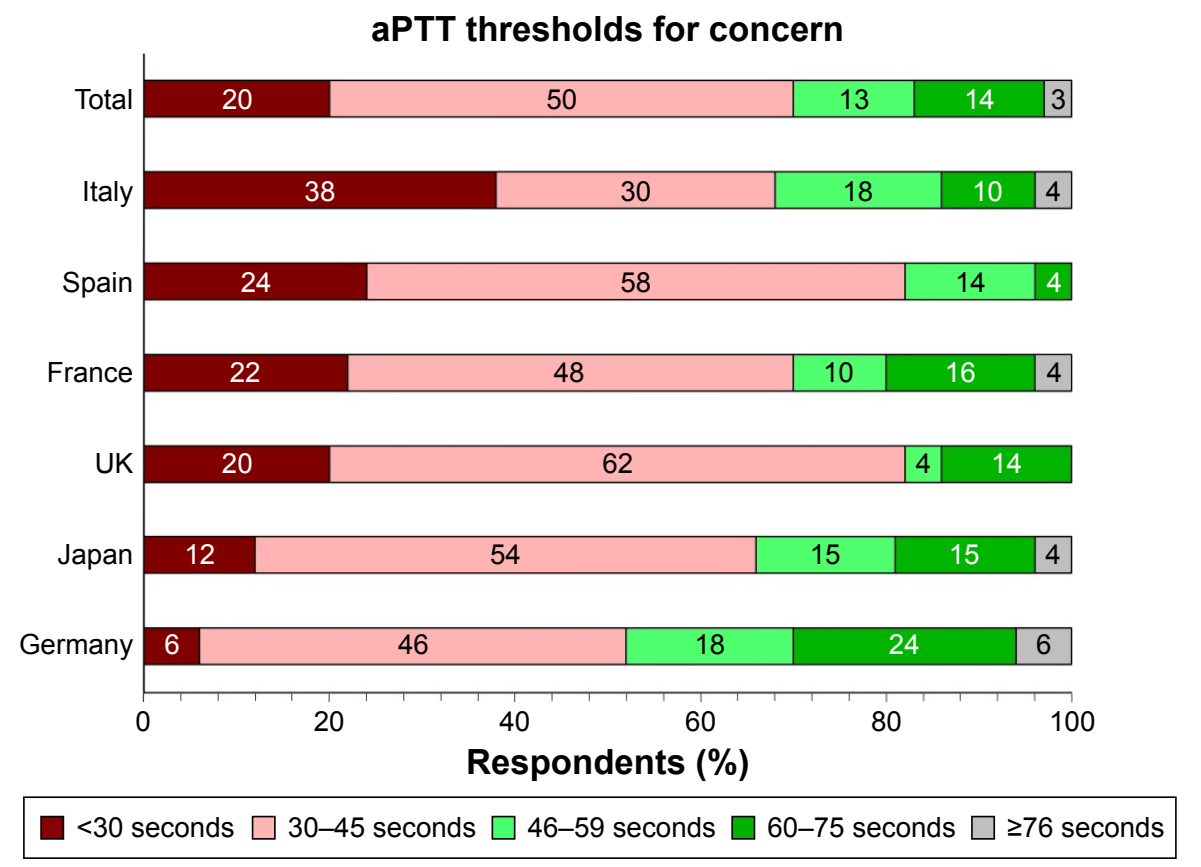

Figure 3 PT (A) and aPTT (B) thresholds for concern.

Abbreviations: aPTT, activated partial thromboplastin time; PT, prothrombin time.

threshold for defining massive PPH, with $64 \%$ of US respondents defining massive $\mathrm{PPH}$ as $>2,000 \mathrm{~mL}$ blood loss. Comparatively, $39 \%$ of the overall global sample defined massive PPH as $>2,000 \mathrm{~mL}$ blood loss, while a roughly equal percentage (34\%) used a threshold of $>1,500 \mathrm{~mL}$. Similar percentages of the overall global and US samples reported using packed red blood cells and fresh frozen plasma for PPH, but nearly half of the overall global sample used clotting factor(s), compared with only one-quarter of the US sample. Cryoprecipitate was used by more physicians in the US sample (74\%) than in the overall global sample (31\%).

Similar to the US survey, the most common threshold for checking coagulation studies was ongoing visible bleeding. Whereas fewer than half of respondents in the US survey selected any other threshold for checking coagulation studies, nearly $60 \%$ of participants in the global survey 
Table I Discovery of bleeding disorders and experience with acquired hemophilia among obstetrician-gynecologists

\begin{tabular}{lllll}
\hline & $\begin{array}{l}\text { Discovered } \\
\text { an underlying } \\
\text { bleeding disorder }\end{array}$ & & \multicolumn{2}{l}{$\begin{array}{l}\text { Encountered } \\
\text { acquired } \\
\text { hemophilia* }\end{array}$} \\
\cline { 2 - 5 } & $\mathbf{N}$ & $\%$ & & n \\
\hline Total & 302 & 58 & 175 & 23 \\
UK & 50 & 56 & 28 & 18 \\
France & 50 & 52 & 26 & 19 \\
Germany & 50 & 70 & 35 & 43 \\
Italy & 50 & 62 & 31 & 32 \\
Spain & 50 & 74 & 37 & 14 \\
Japan & 52 & 35 & 18 & 0 \\
\hline
\end{tabular}

Note: *Among obstetrician-gynecologists who have discovered an underlying bleeding disorder.

identified tachycardia and an estimated blood loss of $1,000 \mathrm{~mL}$ as additional triggers for ordering coagulation studies in this setting. Nearly all respondents in both the global and US surveys reported that coagulation studies were performed on-site at their practice locations, indicating that the availability of such testing was likely not a barrier towards obtaining results.

The interpretation of coagulation test results was an additional factor assessed in this survey. Overall, the PT and aPTT thresholds most commonly identified as indicating concern (13 to 20 seconds and 30 to 45 seconds, respectively), were consistent with reference ranges (typically 10 to 12 seconds for PT and 24 to 37 seconds for aPTT), ${ }^{20,21}$ although reference ranges are known to be variable and depend on the laboratory performing the test, due in part to variability in the reagents, analyzer instruments, and calibration. Outliers regarding PT thresholds included the German cohort, half of whom selected a higher range of 21 to 40 seconds as cause for concern, and the French cohort, the greatest percentage $(40 \%)$ of whom identified a very high threshold of $\geq 50$ seconds. For aPTT, the most significant outlier was the Italian cohort, the highest percentage (38\%) of whom identified a low threshold of $\leq 30$ seconds.

Ensuring the appropriate handling of abnormal test results is an important aspect of diagnosis and was also addressed in the survey. Respondents reported that the majority of abnormal coagulation studies they had ordered in the preceding 5 years were flagged and that normal reference ranges were provided; however, they reported little familiarity with 1:1 mixing studies, tests that would be routinely performed to determine whether a prolonged PT or aPTT was the result of a factor deficiency (adding normal plasma corrects the PT/aPTT) or an inhibitor (adding normal plasma does not correct the PT/aPTT). ${ }^{22}$ Furthermore, in contrast to coagulation studies, on-site mixing studies were not known to be widely available for the survey participants; only $22 \%$ reported that mixing studies were performed at their respective practice locations.

Similar high percentages of participants in the global and US surveys ( $58 \%$ and $52 \%$, respectively) reported having discovered an underlying bleeding disorder in a patient with $\mathrm{PPH}$. Incidence of massive PPH resulting in coagulopathy (acquired or congenital) is reported to be between $0.15 \%$ and $0.5 \%,{ }^{4,23-25}$ with massive blood loss due to uterine atony being the most common cause (39\%). The most common congenital bleeding disorder is VWD, with an estimated prevalence of up to $1 \%$ to $2 \%$ in the population; incidence of $\mathrm{PPH}$ in women with VWD is 1.5 times higher than in controls (6\% vs $4 \%$ ) and transfusion is 4.7 times more likely. ${ }^{26}$ Thus, it might be expected that most obstetricians experienced with PPH related to underlying bleeding disorders will have experience with common congenital and acquired disorders. In contrast, a higher percentage had encountered the rare disorder of acquired hemophilia than might be expected, and also in comparison with the corresponding subset of participants in the US survey ( $23 \%$ and $4 \%$, respectively). However, self-reported knowledge of acquired hemophilia was low, with $80 \%$ of the overall global sample reporting that they knew little or nothing about this rare $(\sim 1$ in $1-1.5$ million) condition characterized by autoantibodies to factor VIII in older adults and postpartum women (up to 6 to 12 months post-delivery). ${ }^{27}$

Higher percentages of respondents overall, and in most countries in the global survey, had consulted a hematologist compared with those in the US survey, although the greatest percentage had done so only once or twice in their careers. An exception to the low rates of hematology consultation was the UK cohort practicing under a national health system with centralized hematology laboratory services; the majority of UK respondents had consulted a hematologist 5 or more times for PPH. Nearly one-quarter of participants overall had never consulted a hematologist for a patient with PPH.

A limitation of this survey was that it did not assess potential systemic differences across countries that may have influenced participants' responses. Additional potential limitations include the elective nature of participation (those with an interest in coagulation studies may have been more likely to respond) and small sample sizes within individual countries.

Nonetheless, these data highlight opportunities for improving awareness of how to rapidly and efficiently identify coagulation disorders among women with PPH. Greater consistency in approaches towards performing and 
interpreting basic laboratory assessments (PT/aPTT), as well as deciding when to consult with a hematologist, may encourage greater focus on identifying the underlying causes of severe PPH.

\section{Conclusion}

Confirming and expanding upon the findings of the similar US survey, the results of this quantitative global survey of obstetrician-gynecologists across different health care delivery systems revealed potential barriers to identifying congenital and acquired bleeding disorders as a contributing factor in PPH. Potential barriers include the lack of a consensus definition for massive $\mathrm{PPH}$, inconsistent ordering and interpretation of basic coagulation studies (PT/aPTT), limited availability of follow-up laboratory tests (mixing studies, factor assays, inhibitor tests), lack of experience with and knowledge of bleeding disorders, and infrequent involvement of a hematologist in the care of patients with PPH. Addressing such barriers in local practice protocols and in national consensus guidelines may serve to facilitate prompt identification of both common and uncommon contributing acquired or congenital bleeding disorders in patients with $\mathrm{PPH}$ and provide valuable information for ensuring appropriate therapeutic management.

\section{Acknowledgments}

The quantitative and qualitative research surveys described in this manuscript were completed by Harris Interactive, New York, NY, USA, with funding provided by Novo Nordisk Healthcare AG., Zurich, Switzerland. Editorial assistance was provided by Anna Abt, PhD, of ETHOS Health Communications in Yardley, PA, USA, with financial support from Novo Nordisk Inc., in compliance with international guidelines for Good Publication Practice.

\section{Disclosure}

David L Cooper is an employee of Novo Nordisk Inc. Andra H James and Michael J Paidas report no conflicts of interest in this work.

\section{References}

1. Bonnet MP, Deneux-Tharaux C, Bouvier-Colle MH. Critical care and transfusion management in maternal deaths from postpartum haemorrhage. Eur J Obstet Gynecol Reprod Biol. 2011;158(2):183-188.

2. Borna S, Hantoushzadeh S. Acquired hemophilia as a cause of primary postpartum hemorrhage. Arch Iran Med. 2007;10(1):107-110.

3. Brace V, Kernaghan D, Penney G. Learning from adverse clinical outcomes: major obstetric haemorrhage in Scotland, 2003-2005. Br J Obstet Gynaecol. 2007;114(11):1388-1396.

4. James AH, McLintock C, Lockhart E. Postpartum hemorrhage: When uterotonics and sutures fail. Am J Hematol. 2012;87(S1):S16-S22.
5. Sheikh L, Najmi N, Khalid U, Saleem T. Evaluation of compliance and outcomes of a management protocol for massive postpartum hemorrhage at a tertiary care hospital in Pakistan. BMC Pregnancy Childbirth. 2011;11:28

6. Wise A, Clark V. Challenges of major obstetric haemorrhage. Best Pract Res Clin Obstet Gynaecol. 2010;24(3):353-365.

7. Montufar-Rueda C, Rodriguez L, Jarquin JD, et al. Severe postpartum hemorrhage from uterine atony: a multicentric study. J Pregnancy. 2013; 2013:525914.

8. Silver RM, Major H. Maternal coagulation disorders and postpartum hemorrhage. Clin Obstet Gynecol. 2010;53(1):252-264.

9. Collins PW, Hirsch S, Baglin TP, et al; UK Haemophilia Centre Doctors' Organisation. Acquired hemophilia A in the United Kingdom: a 2-year national surveillance study by the United Kingdom Haemophilia Centre Doctors' Oganisation. Blood. 2007;109(5):1870-1877.

10. Kessler CM, Ludlam CA. The treatment of acquired factor VIII inhibitors: worldwide experience with porcine factor VIII concentrate. International Acquired Hemophilia Study Group. Semin Hematol. 1993; 30(2 Suppl 1):22-27.

11. Lak M, Sharifian RA, Karimi K, Mansouritorghabeh H. Acquired hemophilia A: clinical features, surgery and treatment of 34 cases, and experience of using recombinant factor VIIa. Clin Appl Thromb Hemost. 2010;16(3):294-300.

12. Morrison AE, Ludlam CA, Kessler C. Use of porcine factor VIII in the treatment of patients with acquired hemophilia. Blood. 1993;81(6): $1513-1520$.

13. Paidas MJ, Hossain N. Unexpected postpartum hemorrhage due to an acquired factor VIII inhibitor. Am J Perinatol. 2014;31(8):645-654.

14. Tengborn L, Baudo F, Huth-Kühne A, et al. Pregnancy-associated acquired haemophilia A: results from the European Acquired Haemophilia (EACH2) registry. Br J Obstet Gynaecol. 2012;119(12): $1529-1537$.

15. James AH. More than menorrhagia: a review of the obstetric and gynaecological manifestations of bleeding disorders. Haemophilia. 2005;11(4):295-307.

16. Ma AD, Kessler CM, Al-Mondhiry HA, Gut RZ, Cooper DL. Use of recombinant activated factor VII for acute bleeding episodes in acquired hemophilia: final analysis from the Hemostasis and Thrombosis Research Society Registry acquired hemophilia study. Blood Coagul Fibrinolysis. 2016;27(7):753-760.

17. Ma AD, Kessler CM, Al-Mondhiry HA, Gut RZ, Cooper DL. US experience with recombinant factor VIIa for surgery and other invasive procedures in acquired haemophilia: analysis from the Hemostasis and Thrombosis Research Society Registry. Haemophilia. 2016;22(1): e18-e24.

18. James AH, Cooper DL, Paidas MJ. Hemostatic assessment, treatment strategies, and hematology consultation in massive postpartum hemorrhage: results of a quantitative survey of obstetrician-gynecologists. Int $J$ Womens Health. 2015;7:873-881.

19. Reding MT, Cooper DL. Barriers to effective diagnosis and management of a bleeding patient with undiagnosed bleeding disorder across multiple specialties: results of a quantitative case-based survey. J Multidiscip Healthc. 2012;5:277-287.

20. Rehman HU. Elevated APTT? How best to follow up. J Fam Pract. 2013;62(6):E1-E3.

21. Tripodi A, Lippi G, Plebani M. How to report results of prothrombin and activated partial thromboplastin times. Clin Chem Lab Med. 2016; 54(2):215-222

22. Kamal AH, Tefferi A, Pruthi RK. How to interpret and pursue an abnormal prothrombin time, activated partial thromboplastin time, and bleeding time in adults. Mayo Clin Proc. 2007;82(7):864-873.

23. Lenox C, Marr L. Scottish Confidential Audit of Severe Maternal Morbidity, 7th Annual Report. Edinburgh, Scotland: Healthcare Improvement Scotland; 2011:1-42.

24. Bateman BT, Berman MF, Riley LE, Leffert LR. The epidemiology of postpartum hemorrhage in a large, nationwide sample of deliveries. Anesth Analg. 2010;110(5):1368-1373. 
25. James AH, Paglia MJ, Gernsheimer T, Grotegut C, Thames B. Blood component therapy in postpartum hemorrhage. Transfusion. 2009;49(11) 2430-2433.

26. James AH, Jamison MG. Bleeding events and other complications during pregnancy and childbirth in women with von Willebrand disease. J Thromb Haemost. 2007;5(6):1165-1169.
27. Collins P, Budde U, Rand JH, Federici AB, Kessler CM. Epidemiology and general guidelines of the management of acquired haemophilia and von Willebrand syndrome. Haemophilia. 2008;14(Suppl 3):49-55.

International Journal of Women's Health

\section{Publish your work in this journal}

The International Journal of Women's Health is an international, peerreviewed open-access journal publishing original research, reports, editorials, reviews and commentaries on all aspects of women's healthcare including gynecology, obstetrics, and breast cancer. The manuscript management system is completely online and includes a very quick and fair peer-review system, which is all easy to use. Visit http://www.dovepress.com/testimonials.php to read real quotes from published authors.

Submit your manuscript here: http://www.dovepress.com/international-journal-of-womens-health-journal 